\title{
Neue Hoffnung im Kampf gegen Tumoren - dank hochintensivem fokussiertem Ultraschall (HIFU)
}

\author{
Bericht zur DEGUM-Pressekonferenz am 5. Dezember 2018 in Berlin
}

Ob in der Gynäkologie, der Inneren Medizin oder der Orthopädie - das Ultraschallverfahren wird in erster Linie zu diagnostischen Zwecken eingesetzt. Doch bei einigen Tumorerkrankungen wird es mittlerweile auch für therapeutische Zwecke angewendet. So können bösartige, inoperable Bauchspeicheldrüsen- und Leberkarzinome beispielsweise mit dem hochintensiven fokussierten Ultraschall (HIFU) effektiv behandelt werden. Patienten müssen sich dafür keiner Operation unterziehen und erleiden kaum Schmerzen. Wie funktioniert der HIFU und auf welche Weise verringert er das Tumorvolumen? Bei welchen Erkrankungen wird er eingesetzt? Antworten auf diese und weitere Fragen gaben Experten der Deutschen Gesellschaft für Ultraschall in der Medizin e. V. (DEGUM) am 5. Dezember 2018 auf einer Pressekonferenz in Berlin.

Zu Beginn der Veranstaltung berichtete DEGUM-Expertin Priv.-Doz. Dr. Dr. Milka Marinova aus der Radiologischen Universitätsklinik Bonn über den Einsatz des HIFU beim inoperablen Bauchspeicheldrüsenkarzinom und darüber, warum das Verfahren durch den Tumor bedingte Schmerzen deutlich lindern und das Tumorvolumen verkleinern kann. „Die Lebensqualität der Bauchspeicheldrüsenkrebs-Patienten kann durch den Tumorschmerz deutlich bis massiv einge- schränkt werden“, so Marinova. „Im Rahmen standardisierter Untersuchungen haben wir herausgefunden, dass eine Behandlung mittels HIFU bei mehr als 80 Prozent der Patienten zu einer wirksamen und anhaltenden Linderung der Tumorschmerzen führt." Sowohl die Schmerzintensität als auch die -empfindung seien stark reduziert worden. Viele Patienten hätten bereits in der ersten Woche nach dem Behandlungsbeginn eine deutliche Verbesserung gespürt. „Bei der Hälfte der initial mit Schmerzmitteln behandelten Patienten war nach 6 Wochen sogar keinerlei Schmerzmedikation mehr erforderlich“, berichtet die Expertin.

\section{Primäre Leberkarzinome - vielver- sprechend mittels HIFU therapieren}

Auch primäre Leberkarzinome können mit dem HIFU-Verfahren behandelt werden. „Mit dem therapeutischen Ultraschall können im Gegensatz zu anderen Behandlungsoptionen auch Lebertumoren in unmittelbarer Nähe zu Gefäßen behandelt werden“, sagt Professor Dr. Holger Strunk, Oberarzt in der Radiologischen Universitätsklinik Bonn. Denn bei dem therapeutischen Ultraschallverfahren müssen keine Nadeln, Sonden oder Ähnliches eingebracht werden. Daher besteht auch keine Gefahr einer Blutung oder der Tumorzellverschleppung, wie sie bei anderen Maßnahmen durch den Stichkanal vorkommen kann. Im Gegensatz zum diagnostischen
Ultraschall werden beim HIFU viel höhere Energien erzeugt. Die Ultraschallwellen sind hier gebündelt und können exakt auf ein nur wenige Millimeter großes Gebiet fokussiert werden. „Es entstehen Temperaturen von mehr als 80 Grad Celsius, wodurch Tumorzellen effektiv abgetötet werden“, erläutert Strunk.

\section{Gutartige Knoten in der Brust schmerzarm und ohne Operation behandeln - HIFU macht's möglich}

Neben bösartigen Tumorerkrankungen können auch gutartige Veränderungen mit dem therapeutischen Ultraschallverfahren behandelt werden - beispielsweise Wucherungen in der Gebärmutter (Myome) und symptomatische Knoten in der Brust, meist Fibroadenome. Der Erfolg der Methode zur Behandlung von Fibroadenomen konnte bereits in zahlreichen internationalen Studien nachgewiesen werden. „Die Größe der Knoten konnte bei der Mehrheit der Betroffenen deutlich reduziert werden - und zwar mit langfristiger Wirkung“, betont Dr. Bettina Böer vom Departement für Frauengesundheit am Universitätsklinikum Tübingen. „Zudem zeigten rund 2 Drittel der Patientinnen mit Fibroadenomen der Brust 1 Jahr nach der Behandlung keine Schmerzen mehr." Außerdem wurden die vitalen Zellen des Fibroadenoms bei zahlreichen Patientinnen effektiv zerstört. Vorteile sind darüber hinaus, dass die Behandlung 
schmerzarm in Lokalanästhesie erfolgen kann und dass - im Gegensatz zu allen anderen Behandlungsverfahren - keine äußere Narbe sichtbar ist.

Knochen- und Weichteiltumoren gezielt verkleinern - warum der fokussierte Ultraschall besonders erfolgsversprechend ist

Zum Schluss der Pressekonferenz referierte Professor Dr. Holger Strunk über den Ein- satz des HIFU bei Knochen- und Weichteiltumoren. „Der Vorteil des therapeutischen Ultraschallverfahrens besteht bei Weichteiltumoren - wie etwa bei sogenannten Desmoiden - darin, dass es nach der Behandlung bei einem Großteil der Patienten nicht zu einem Rückfall in der behandelten Region kommt“, so Strunk. „Das liegt daran, dass der HIFU im Gegensatz zu anderen abtragenden und operativen Verfahren quasi berührungsfrei funktioniert.“ Zudem konnten erste Fallberichte zeigen, dass es nach der HIFU-Behandlung bei etwa 75 Prozent der Patienten zu einer deutlichen Größenreduktion der Tumoren und zur Symptomlinderung kam. 